\title{
Hierarchical micro-/macroporous TS-1 zeolite epoxidation catalyst prepared by steam assisted crystallization
}

\author{
Valentin Smeets ${ }^{\text {a }}$, Eric M. Gaigneaux ${ }^{\text {a }}$, Damien P. Debecker ${ }^{\text {a,* }}$ \\ ${ }^{a}$ Institute of Condensed Matter and Nanosciences (IMCN), UCLouvain, Place Louis Pasteur, 1, Box L4.01.09, 1348 Louvain- \\ la-Neuve, Belgium.*E-mail: damien.debecker@uclouvain.be
}

\begin{abstract}
:
Mesoporous $\mathrm{Ti}-\mathrm{SiO}_{2}$ nanoparticles were prepared under alkaline conditions in the presence of a surfactant and were subsequently converted into a hierarchical micro-/macroporous TS-1 zeolite with large crystal size using steam assisted crystallization. In this procedure, the precursor nanoparticles were used both as macroporous hard template and as $\mathrm{Si}$ and $\mathrm{Ti}$ sources. The secondary macroporosity is a reminiscence of the nanoparticles which undergo dissolution and recrystallization upon steaming. The obtained catalyst has structural properties comparable to benchmark TS-1. We show that the successful conversion of the amorphous material into a fully crystalline catalyst stands for its excellent catalytic performance in aqueous media. Besides, the combination of large crystal size with a hierarchical pore structure ensures easy catalyst handling and processing without compromising on the catalytic activity.
\end{abstract}

Keywords: hierarchically porous zeolite, TS-1, macroporous material, steam assisted crystallization, epoxidation 


\section{Introduction}

Titanium silicalite-1 (TS-1) zeolite [1] is an important functional material that has been used for the past decades as a selective oxidation catalyst, e.g. in olefin epoxidation [2]. While preserving the MFI type structure that ensures its remarkable activity, it is desirable to design new TS-1 based catalysts for specific catalytic applications and reactor configurations.

On the one hand, catalytic performance is strongly limited by the slow diffusion of reactants and products in the micropores of the zeolite [3-6]. In some cases, the molecules cannot enter the microporosity and are thus restrained to the external surface. Therefore, strategies are put in place to enhance the surface-tovolume ratio of the catalyst, i.e. to reduce the diffusion path length within the inorganic walls and/or to increase the external surface area [4]. Thus, TS-1 can be shaped [7] as nano-sheets [8], pillared materials [9], membranes [10], etc. Another way out is to add a secondary porosity [11-14], with benefits in terms of active site accessibility. This was previously reported for micro-/mesoporous TS-1 [15-17], but the strategy was also shown to be applicable to micro-/macroporous TS-1 [18] as well as three level micro-/meso/macroporous structures [19-21] and hollow TS-1 crystals [22,23]. More simply, the crystals size can be reduced in order to increase the catalytic performance.

On the second hand, the handling of small zeolite nanocrystals is complicated because such colloidal objects are refractory to mechanical recovery by filtration or decantation. Zeolite nanocrystals cannot be implemented in fixed bed flow reactor as their packing would result in an excessive pressure drop. Therefore, another objective of the modification of TS- 1 materials is to obtain larger objects which fully benefit from the advantages of heterogeneous catalysts in terms of recoverability and reusability. Thus, TS- 1 material can be prepared in the form of hierarchically porous microparticles $[24,25]$ or even self-standing monoliths [26]. Alternatively, the nanosized crystals can be dispersed onto a porous support, e.g. diatomites [27].

Considering the above, it is highly desirable to obtain large crystals while preserving a high surface-tovolume ratio. The Steam Assisted Crystallisation (SAC) method, also called Dry Gel Conversion (DGC), has become a popular procedure to prepare hierarchically porous zeolites with a good control on texture and particle size [28-32]. In this method, an amorphous (mixed) oxide is prepared in the form of a dry gel and treated under hydrothermal conditions in the presence of a structure-directing agent and of steam. Upon steaming, the crystallization of the zeolite proceeds via an oriented attachment route, which consists in the aggregation and alignment of tiny crystallites that form bigger crystals [29]. This technique therefore differs from the hydrothermal synthesis of zeolites, the latter involving the crystallization route by nucleation and growth in liquid phase water. The particle size can be tuned by varying the temperature and crystallization time, whereas the porosity is typically controlled in the mesoporous range by the addition of templates $-e . g$. organosilanes - that prevent the fusion of the crystallites, or by leveraging on the grinding and drying steps of the gel prior to autoclaving [32]. Alternatively, Schwieger and co-workers showed that mesoporous silica and aluminosilicate nanoparticles could be used both as hard template and precursors in the synthesis of micro-/macroporous silicalite and ZSM-5 by SAC, respectively $[33,34]$.

Herein, we propose to combine titanium and silicon precursors in the synthesis of amorphous $\mathrm{Ti}-$ $\mathrm{SiO}_{2}$ nanoparticles and to use the latters in the preparation of hierarchical TS-1 material featuring both a micro-/macroporosity and large crystal size. To the best of our knowledge, the incorporation of titanium in such precursor material and its further crystallization into fully functional hierarchical TS- 1 catalyst by SAC has never been reported. We aim to demonstrate that controlling the incorporation of titanium during the preparation of the precursor particles prior to the thermal treatment allows obtaining TS- 1 presenting both proper Ti speciation in the MFI-type structure and a secondary intracrystalline macroporosity. The successful conversion of the amorphous material into crystalline TS-1 is exploited in the catalytic conversion of allyl alcohol into glycidol in water, and compared to the benchmark microporous TS-1 catalyst.

\section{Experimental}

\subsection{Preparation of the materials}

\subsubsection{Preparation of the reference catalyst}

Benchmark TS-1 ('TS-1') was prepared with a Ti loading of $1.8 \%$ (here and after the loading is expressed as $\mathrm{mol} \mathrm{Ti} /(\mathrm{mol} \mathrm{Ti}+\mathrm{mol} \mathrm{Si}) \times 100 \%)$ using titanium isopropoxide (TiiP) and tetraethyl orthosilicate (TEOS) as $\mathrm{Ti}$ and $\mathrm{Si}$ sources, respectively, and tetrapropylammonium hydroxide (TPAOH) as structure directing agent [35]. The detailed preparation procedure is available in the Electronic Supplementary Information. 


\subsubsection{Preparation of the hierarchical micro- /macroporous TS-1}

The micro-/macroporous TS-1 (denoted as 'TS1_MAC') was prepared by adapting the procedure of Machoke et al. for the preparation of macroporous silicalite [33]. First, mesoporous $\mathrm{Ti}-\mathrm{SiO}_{2}$ particles, denoted 'MSTP', were synthesized as follows: $1 \mathrm{~g}$ of hexadecyltrimethylammonium bromide (CTAB, Alfa Aesar, 98\%) was added to $140 \mathrm{ml}$ distilled $\mathrm{H}_{2} \mathrm{O}$ and 480 $\mathrm{ml} \mathrm{EtOH}$ under stirring. After $10 \mathrm{~min}$ stirring, $24 \mathrm{ml}$ $\mathrm{NH}_{4} \mathrm{OH} 25 \%$ wt. aq. (Merck) was added (Solution A). In a separate vessel, $3.130 \mathrm{~g}$ TEOS and $0.097 \mathrm{~g}$ titanium butoxide (1.8\% at. Ti) (TiBuO, Sigma-Aldrich, 97\%) were mixed in $5 \mathrm{ml} \mathrm{EtOH}$ under vigorous stirring and then stirred for $1 \mathrm{~h}$ at room temperature (Solution B). The pre-mixing of the $\mathrm{Si}$ and $\mathrm{Ti}$ precursors was essential to ensure the incorporation of titanium into the silica nanoparticles. Thereafter, solution B was added dropwise to solution A, resulting in a clear yellowish solution. After $10 \mathrm{~min}$ stirring at room temperature, a white precipitate appeared and the solution was further stirred for $2 \mathrm{~h}$. The precipitate was subsequently recovered by filtration, washed with distilled $\mathrm{H}_{2} \mathrm{O}$ and dried overnight at $75^{\circ} \mathrm{C}$. Finally, CTAB was removed by calcination at $550^{\circ} \mathrm{C}$ for $6 \mathrm{~h}\left(5^{\circ} \mathrm{C} / \mathrm{min}\right)$. In the second step, $250 \mathrm{mg}$ of MSTP was impregnated with $0.34 \mathrm{ml}$ $40 \%$ wt. TPAOH (TPAOH/ $/ \mathrm{SiO}_{2}$ molar ratio of 0.16 ) in a crucible and homogenised with a spatula until a homogeneous paste was obtained $\left(\mathrm{V}_{\mathrm{MSTP}} /\left(\mathrm{V}_{\mathrm{MSTP}}+\right.\right.$ $\left.\left.\mathrm{V}_{\text {TPAOH }}\right)=0.74\right)$. The mixture was then put to rest for 24h. After that time, the solidified paste was crushed manually into small pieces and transferred into a $70 \mathrm{ml}$ teflon-lined stainless steel autoclave containing $20 \mathrm{ml}$ of distilled $\mathrm{H}_{2} \mathrm{O}$ in a separate vessel. After 96h SAC at $130^{\circ} \mathrm{C}$, the solid was successively washed with distilled water and $\mathrm{EtOH}$ so as to facilitate drying without altering the porosity. It was then dried at $75^{\circ} \mathrm{C}$ overnight and finally calcined at $550^{\circ} \mathrm{C}$ for $5 \mathrm{~h}$ $\left(5^{\circ} \mathrm{C} / \mathrm{min}\right)$.

\subsection{Characterization of the materials}

The Ti content of the materials was measured by ICP-AES on an ICP 6500 instrument (Thermo Scientific Instrument) after dissolution of the samples by sodium peroxide fusion. XPS experiments were carried out using an SSX 100/206 spectrometer (Surface Science Instruments, USA) with Al-K $\alpha$ radiation operated at $10 \mathrm{kV}$ and $20 \mathrm{~mA}$. The binding energy scale was calibrated on the Si $2 p$ peak, fixed at $103.5 \mathrm{eV}$ [36]. The quantification of $\mathrm{Ti}$ in $\mathrm{Ti}-\mathrm{O}-\mathrm{Si}$ and Ti-O-Ti was based on the decomposition of the $2 \mathrm{p}_{3 / 2}$ peak at approximately 460.0 and $458.5 \mathrm{eV}$, respectively $[37,38]$. Si was quantified on the basis of the Si $2 p$ peak at $103.5 \mathrm{eV}$ [36]. Powder X-ray diffraction (PXRD) patterns were recorded at room temperature with a D8 ADVANCE Bruker diffractometer equipped with a LYNXEYE XE-T detector, using copper $\mathrm{K} \alpha$ radiation $(\lambda=1.5418 \AA)$ in a $2 \theta$ range of $5-80^{\circ}$ (step $=0.02^{\circ}$, time per step $=0.15 \mathrm{~s}$ ). The $\mathrm{X}$-ray source was operated with a tension of $40 \mathrm{kV}$ and a current of $30 \mathrm{~mA}$. For display, the diffractograms were normalized by the most intense diffraction peak. The DR UV-VIS spectra were recorded on a Shimadzu UV-3600 Plus UV-Vis-NIR Spectrophotometer with a Harrick single-beam Praying Mantis Diffuse Reflectance collection system. The spectra were recorded at room temperature in 20000 $50000 \mathrm{~cm}^{-1}$ range. A Spectralon ${ }^{\circledR}$ Diffuse Reflectance Standard was used to measure the background spectra. The DR UV-VIS spectra were background corrected, normalized by the most intense absorption band, and the Kulbelka Munk function was used to display the data. Scanning electron microscopy (SEM) images were taken using a JEOL $7600 \mathrm{~F}$ microscope with a $1-5 \mathrm{kV}$ voltage. Samples were pre-treated with a chromium sputter coating of $15 \mathrm{~nm}$ carried out under vacuum with a Sputter Metal 208 HR (Cressington). SEM-FEG pictures were obtained with a Hitachi SU-70. Textural properties were determined from $\mathrm{N}_{2}$ adsorption/desorption isotherms at $-196^{\circ} \mathrm{C}$ using a Tristar 3000 instrument (Micromeritics, USA). Prior to measurement, the samples were degassed overnight under vacuum at $150^{\circ} \mathrm{C}$. The BET specific surface area of MSTP was evaluated in the $0.05-0.3 \mathrm{P} / \mathrm{P}_{0}$ range. For hierarchical zeolites, the external specific surface area which includes the specific surface area of mesopores was evaluated by the slope of the line drawn in the linear portion of the t-plot in the 3.5-5 $\AA$ thickness range; the micropore volume was given by the intercept. The total pore volume was measured at $\mathrm{P} / \mathrm{P}_{0}=$ 0.98 . The pore size distribution was obtained from the adsorption branch using the BJH method.

\subsection{Catalytic tests}

The catalytic performance was evaluated in water for the epoxidation of allyl alcohol with hydrogen peroxide as the oxidant. The reaction was carried out in a two-necked round-bottomed glass reactor at $45^{\circ} \mathrm{C}$, equipped with a magnetic stirrer and a rubber septum. In a typical run, $0.528 \mathrm{~g}(0.9 \mathrm{M})$ allyl alcohol (Acros Organics, 99\%), $0.037 \mathrm{~g}$ (0.05 M) Butan-1-ol (SigmaAldrich, $\geq 99.4 \%$ ) - used as the internal standard - and $50 \mathrm{mg}\left(5 \mathrm{~g} . \mathrm{L}^{-1}\right)$ catalyst were pre-mixed in $9.152 \mathrm{~g}$ of distilled $\mathrm{H}_{2} \mathrm{O}$ and stirred for $10 \mathrm{~min}$. To start the reaction, $0.204 \mathrm{~g}(0.18 \mathrm{M})$ of $30 \%(\mathrm{w} / \mathrm{w})$ aqueous $\mathrm{H}_{2} \mathrm{O}_{2}$ was added and the mixture was allowed to react for $3 \mathrm{~h}$. The product formation was followed by collecting aliquots at regular time intervals and by analysing them 
in gas chromatography, using a Varian CP-3800 chromatograph equipped with a FID detector and a capillary column (BR-5, $30 \mathrm{~m}, 0.32 \mathrm{~mm}$ i.d., $1.0 \mu \mathrm{m}$ film thickness). Prior to injection, water was removed by an extraction step with ethyl acetate $(50: 50 \mathrm{v} / \mathrm{v})$.

\section{Results and discussion}

The materials were first analysed by PXRD in order to evaluate the bulk phase crystallinity. The diffractogram of the starting material MSTP does not show any peak, confirming the amorphous nature of these $\mathrm{Ti}-\mathrm{SiO}_{2}$ particles. The TS-1_MAC catalyst exhibited the crystalline MFI-type structure identical to TS-1 zeolite (Figure 1a). This result demonstrates that the formation of the zeolitic framework in TS-1_MAC occurred during the steaming step. Importantly, no $\mathrm{TiO}_{2}$ anatase - having a signature peak at $2 \theta=25^{\circ}[39,40]-$ was detected, thereby excluding the presence of large crystalline $\mathrm{TiO}_{2}$ domains in the materials.
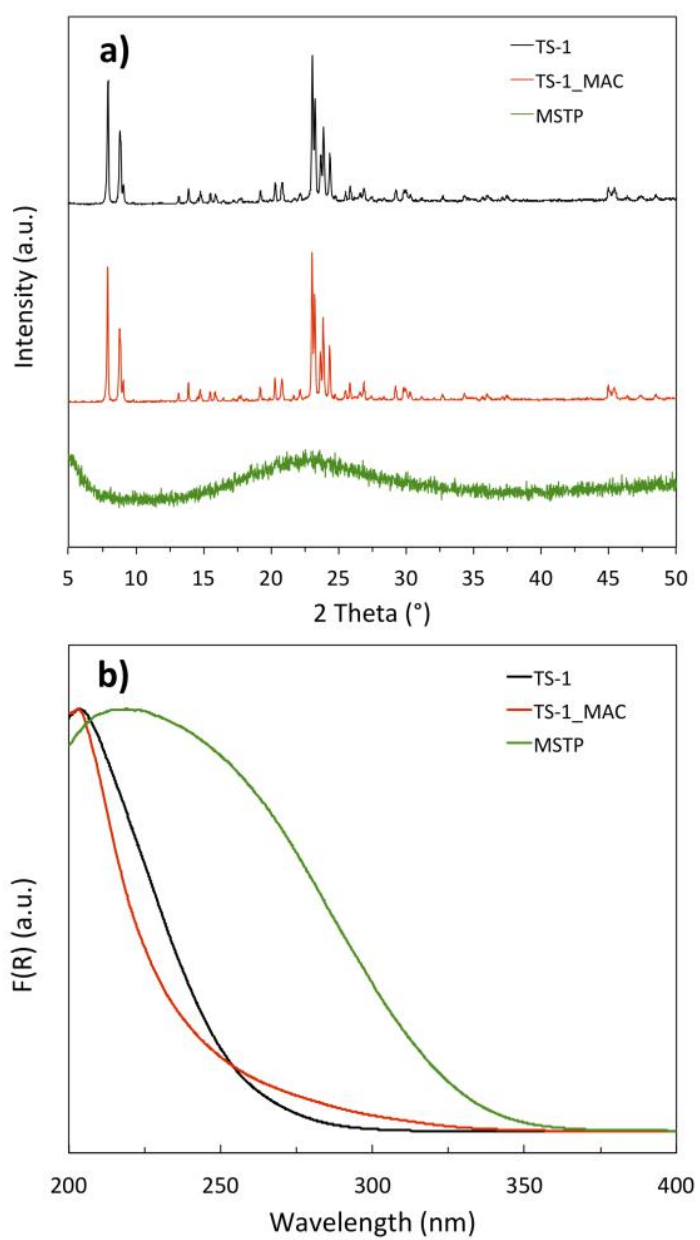

Figure 1. a) Normalized PXRD patterns of the zeolitic catalysts; diffractogram of amorphous MSTP is shown for comparison; b) Normalized DR UV-visible spectra of the catalysts $(\mathrm{F}(\mathrm{R})$ is the Kubelka-Munk function) and of the starting material MSTP.
In order to have deeper insight into the incorporation of titanium in the materials, an elemental analysis was first carried out by ICP-AES and revealed that the experimental $\mathrm{Ti}$ content was respectively of $1.6 \%$ and $1.7 \%$ for TS-1 and TS-1_MAC catalysts (Table 1), thus close to the nominal loading. Further investigations by XPS analysis allowed to determine the $\mathrm{Ti}$ concentration at the surface as well as the quality of the Ti dispersion (Table 1 and Figure S1, ESI). For both zeolitic catalysts, the surface composition matched closely the bulk content, indicating a homogeneous $\mathrm{Ti}$ incorporation throughout the material. On the opposite, the surface $\mathrm{Ti}$ content was very low in the starting material MSTP, highlighting the fact that Ti is poorly dispersed in the amorphous dry gel, with presumably a gradient of concentration and high $\mathrm{Ti}$ content in the bulk of the materials. After applying the SAC procedure, however, a homogeneous zeolitic material is obtained.

Table 1. Percentage of Ti species $(\mathrm{mol} \mathrm{Ti} /(\mathrm{mol} \mathrm{Ti}+\mathrm{mol} \mathrm{Si})$ $\times 100 \%$ ) in the catalysts (bulk composition, ICP-AES) and at the catalyst surface (from XPS)

\begin{tabular}{lllll}
\hline & Bulk Ti \% $^{\mathrm{a}}$ & $\begin{array}{l}\text { Surf. } \\
\text { Ti \% }\end{array}$ & $\begin{array}{l}\text { Surf. } \\
\text { Ti-O-Si \% }\end{array}$ & $\begin{array}{l}\text { \% } \\
\text { O-Si- }^{\mathrm{b}}\end{array}$ \\
\hline TS-1 & $1.6(1.8)$ & 1.3 & 1.2 & 90 \\
TS-1_MAC & $1.7(1.8)$ & 1.7 & 1.0 & 56 \\
MSTP & - & 0.3 & $-^{c}$ & - \\
\hline
\end{tabular}

a Nominal composition calculated from the precise amounts of precursors actually used in each synthesis is given in brackets. ${ }^{\mathrm{b}} \%$ $\mathrm{Ti}-\mathrm{O}-\mathrm{Si}=($ Surf. Ti-O-Si \% / Surf. Ti \% $) \times 100 .{ }^{c}$ The amount of titanium at the surface was too low for this sample to allow a proper decomposition of the Ti $2 p$ peak.

The third column of Table 1 indicates the fraction of Ti species that are truly dispersed in the silica matrix, forming $\mathrm{Ti}-\mathrm{O}-\mathrm{Si}$ bonds, as determined by the decomposition of the $\mathrm{Ti} 2 \mathrm{p}$ peak [41]. The catalyst prepared by $\mathrm{SAC}$ shows a relatively lower $\mathrm{Ti}$ dispersion as compared to TS-1. As no $\mathrm{TiO}_{2}$ anatase was detected by PXRD (Figure 1a), the fraction that is not well incorporated in the silica matrix is assigned to extraframework $\mathrm{Ti}$ species in 5- and 6-coordination states, possibly in the form of Ti oligomers [42]. This is also suggested by the UV-visible spectrum of the TS1_MAC catalyst (Figure 1b), which shows a minor contribution of highly coordinated $\mathrm{Ti}$ species in the region between 250 and $300 \mathrm{~nm}$. Nevertheless, the main absorption band for this catalyst is found in the 200 $210 \mathrm{~nm}$ range - as in TS-1 - which corresponds to highly dispersed tetrahedral $\mathrm{Ti}$ species inside the framework. As a comparison, the XPS spectrum of MSTP shows a Ti $2 p_{3 / 2}$ peak at $458.9 \mathrm{eV}$ (see Figure S1d, ESI), suggesting a major contribution from Ti-O$\mathrm{Ti}$ bonds present in polymeric $\mathrm{Ti}$ oxide species. This result is further supported by the UV-visible spectrum, 
which exhibits a much higher contribution of highly coordinated Ti species for MSTP, which is a clear indication of the poor dispersion of $\mathrm{Ti}$ in this starting material. For this sample, the absorption in the region above $300 \mathrm{~nm}$ may not exclude the presence of $\mathrm{TiO}_{2}$ anatase $[43,44]$. Yet, these clusters would be too small to be detected by PXRD. In any case, the extraframework Ti species are converted into framework Ti species by the SAC treatment [13].
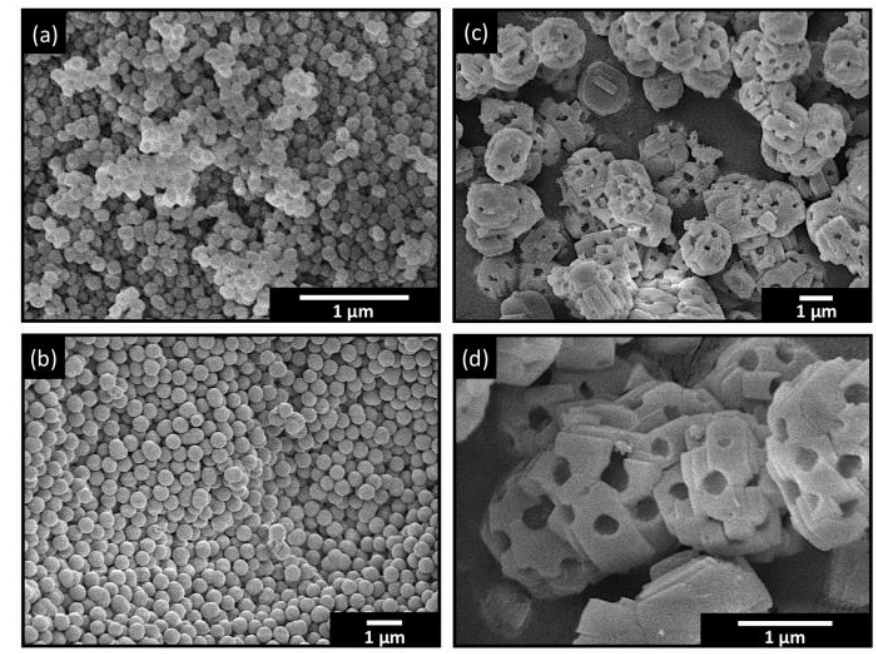

Figure 2. SEM image of a) TS-1, b) MSTP, and c-d) TS1_MAC.

In SEM, TS-1_MAC appeared as holed crystals with size in the $1.5-2 \mu \mathrm{m}$ range (Figure $\mathbf{2 c - d}$ ), thus 15 times larger than the typical nano-sized crystals of TS-1 which show dimensions between 100 and $150 \mathrm{~nm}$ (Figure 2a) [35]. TS-1_MAC crystals are smaller than the Ti-free silicalite-1 crystals prepared by Machoke et al. $(c a .4 \mu \mathrm{m})$ [33], which suggests a lower rate of crystallization in the presence of titanium [35]. This assumption is further supported by the fact that the crystallization did not occur when performing the SAC at $110^{\circ} \mathrm{C}$, as indicated for silicalite-1 [33]. In the present case, the crystallisation step had to be carried out at $130^{\circ} \mathrm{C}$ to obtain the crystalline TS -1 . Each embedded macropore in TS-1_MAC has a pore opening between 200 and $300 \mathrm{~nm}$. These macropores are a reminiscence of the individual MSTP particles (see Figure 2b) which have an average diameter of $c a .450 \mathrm{~nm}$ and serve both as a precursor for the TS-1 crystallization and as a hard template.

From the above, the mechanism of formation of TS-1_MAC crystals can be schematized as represented in Scheme 1. Each crystal is formed by a hard templating route, in which the embedded macropores result from the dissolution-recrystallization of the amorphous MSTP particles upon steaming in the presence of the structure directing agent [33]. During this key step, the poorly dispersed $\mathrm{Ti}$ species in the amorphous precursor particles are incorporated in the zeolitic framework in a 4-coordination mode. The crystals then grow via the oriented attachment of tiny crystallites, following the mechanism proposed by Song et al. [29].

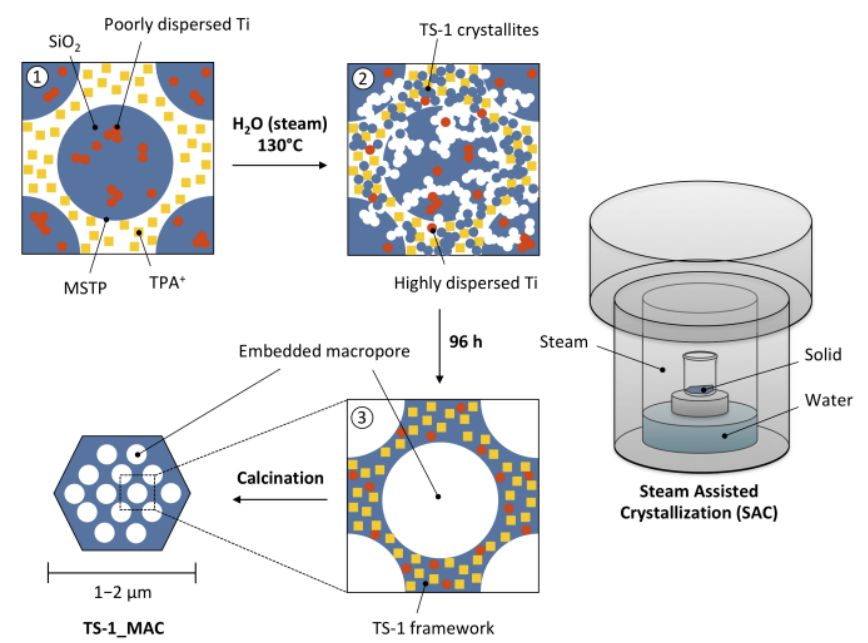

Scheme 1. Proposed mechanism of formation of TS1_MAC. In step 1, the MSTP particles are surrounded by the zeolite structure directing agent. In step (2), the dissolutionrecrystallization proceeds in the presence of the SDA upon steaming to form tiny TS-1 crystallites which aggregate around the precursor particles to form bigger crystals. In step (3), the hierarchical zeolite framework is fully crystallized and displays embedded macropores.

In $\mathrm{N}_{2}$ physisorption, the isotherms of the MSTP solid revealed the presence of supermicropores formed upon templating with $\mathrm{CTAB}$. Yet, no micropores were found on the t-plot. After the SAC treatment, TS1_MAC expectedly showed a Type I isotherm characteristic of microporous solids, and very close to the TS-1 sample (Figure 3). The micropore volume $\mathrm{V}_{\mu}$ is similar in both catalysts (Table 2). Although the macropores of TS-1_MAC fall off the measurment range of the $\mathrm{N}_{2}$ physisorption technique, the forced closure of the isotherm upon desorption at $\mathrm{P} / \mathrm{P}_{0}$ of 0.4 0.5 due to the tensile stress effect [45] suggests also the presence of mesopores smaller than $3.8 \mathrm{~nm}$ in the pore walls of the material, as reported in the literature $[33,46]$ (see also inset of Figure 3). The large $\mathrm{N}_{2}$ uptake at high $\mathrm{P} / \mathrm{P}_{0}$ in this sample is assigned to interparticular voids between the crystals $(\sim 40-50 \mathrm{~nm})$. The external surface area of TS-1 nanosized crystals was of 130 $\mathrm{m}^{2} \cdot \mathrm{g}^{-1}$. Importantly, despite the much larger crystal size of TS-1_MAC, the external surface area reached 150 $\mathrm{m}^{2} \cdot \mathrm{g}^{-1}$ (Table 2), thanks to the surface area generated by the additional porosity. 
Table 2. Textural properties of the catalysts

\begin{tabular}{llll}
\hline & $\begin{array}{l}\mathbf{S}_{\text {ext }} \\
\left(\mathbf{m}^{2} \cdot \mathbf{g}^{-1}\right)^{\mathrm{a}}\end{array}$ & $\begin{array}{l}\mathbf{V}_{\mu} \\
\left(\mathbf{c m}^{3} \cdot \mathbf{g}^{-\mathbf{1}}\right)^{\mathrm{a}}\end{array}$ & $\begin{array}{l}\mathbf{V}_{\mathbf{p}} \\
\left(\mathbf{c m}^{3} \cdot \mathbf{g}^{-1}\right)^{\mathrm{b}}\end{array}$ \\
\hline TS-1_MAC & 130 & 0.15 & 0.28 \\
TS-1_MAC & 150 & 0.14 & 0.23 \\
MSTP & $610^{\mathrm{c}}$ & 0 & 0.40 \\
\hline
\end{tabular}

${ }^{a}$ Calculated from the t-plot. ${ }^{b}$ Measured at $\mathrm{P} / \mathrm{P}_{0}=0.98$. ${ }^{\mathrm{c}}$ For this sample, the external surface area is evaluated by the BET method.

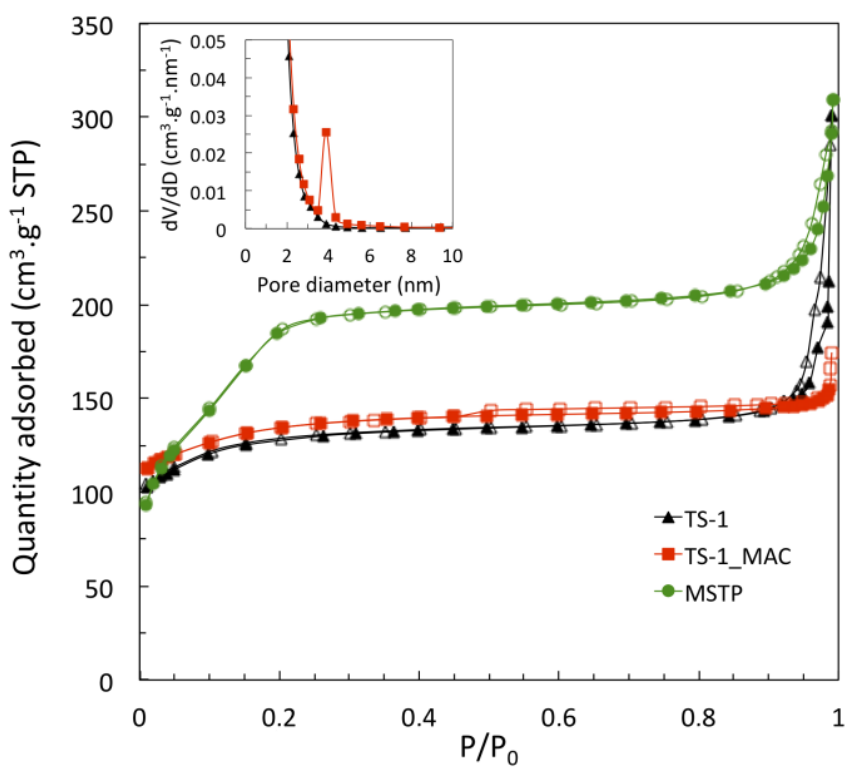

Figure 3. $\mathrm{N}_{2}$ adsorption-desorption isotherms of the materials. Desorption PSDs of TS-1 and TS-1_MAC are shown in inset.

The catalytic performance of TS-1_MAC was evaluated for the epoxidation of allyl alcohol to glycidol in water with $\mathrm{H}_{2} \mathrm{O}_{2}$ as the oxidizing agent. From the kinetic curves presented in Figure $\mathbf{4}$ - showing the glycidol production over time - almost no activity was observed when testing the amorphous MSTP starting material. Indeed, it is well known that amorphous $\mathrm{Ti}-$ $\mathrm{SiO}_{2}$ catalysts are sensitive to the presence of $\mathrm{H}_{2} \mathrm{O}$ molecules [47], leading to strong deactivation issues in the presence of large amounts of water [48]. Besides, XPS and UV-visible spectroscopy showed that Ti was poorly dispersed in the MSTP sample (vide supra). On the opposite, TS-1_MAC was highly active as it showed similar performance as the benchmark TS-1. Thus, starting from an inactive $\mathrm{Ti}-\mathrm{SiO}_{2}$ material, an active epoxidation catalyst is obtained using SAC. The high activity of this material can be ascribed to the successful formation of the MFI crystal structure upon steaming (see Figure 1). Indeed, the incorporation of single site $\mathrm{Ti}$ in the crystalline framework during the dissolution-recrystallization step accounts for the formation of the active sites, and the MFI structure is known to confer an intrinsic resistance towards deactivation in water.

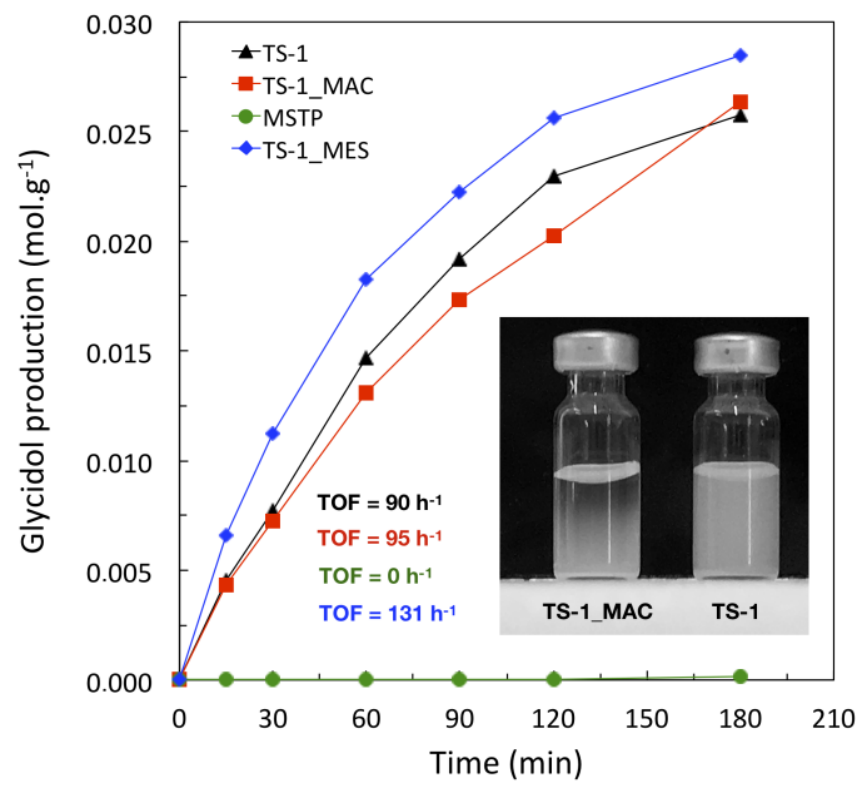

Figure 4. Kinetic data for the conversion of allyl alcohol into glycidol in $\mathrm{H}_{2} \mathrm{O}$ using aqueous solution of hydrogen peroxide as oxidizing agent. The initial turnover frequency (TOF) was approximated by the glycidol production after $15 \mathrm{~min}$ reaction time and normalized by the amount of active $\mathrm{Ti}$ (see "Surf. Ti-O-Si \%" in Table 1). No other products were detected. Experimental conditions: $\mathrm{T}=45^{\circ} \mathrm{C}$, [Allyl alcohol] $=0.9$ mol. ${ }^{-1},\left[\mathrm{H}_{2} \mathrm{O}_{2}\right]=0.18 \mathrm{~mol} . \mathrm{l}^{-1}$. In inset is shown a picture illustrating the fact that the TS-1_MAC sample settles faster than TS-1 in water. The picture was taken $2 \mathrm{~h}$ after sonicating the catalyst suspension $\left(20 \mathrm{~g} . \mathrm{l}^{-1}\right)$ for $30 \mathrm{~min}$.

Both TS-1 and TS-1_MAC exhibit comparable performance, not only in terms of glycidol production and full selectivity towards the epoxide [49], but also as regard to their turnover frequency (TOF). Yet, increasing the particle size gives a competitive advantage by reducing the energy cost for separation by facilitating the catalyst recovery by centrifugation or filtration. For example, the sedimentation behaviour was qualitatively assessed for both TS-1_MAC and TS-1 and we showed that the former could be separated much faster (see inset of Figure 4). Zuo et al. have shown, however, that the turnover frequency decreases when increasing the crystal size of non-hierarchical TS1 , because the external surface areas was lowered $(85$ and $145 \mathrm{~m}^{2} \cdot \mathrm{g}^{-1}$ for crystal size of 1200 and $200 \mathrm{~nm}$, respectively) [50]. In our system, the key factor is that the additional porosity of TS-1_MAC allows to overcome this limitation. While switching from $\sim 100$ nm TS-1 particles to $\sim 1500 \mathrm{~nm}$ crystals of TS-1_MAC, a high external surface area is maintained $\left(S_{\text {ext }}=130\right.$ $\mathrm{m}^{2} \cdot \mathrm{g}^{-1}$ and $150 \mathrm{~m}^{2} \cdot \mathrm{g}^{-1}$ respectively).

The beneficial effect of the introduction of a hierarchical porosity in TS-1 on the catalytic performance was previously reported for micro/mesoporous TS-1. In order to compare TS-1_MAC to 
such type of catalyst, a micro-/mesoporous TS-1 denoted TS-1_MES - was synthesized with the same structure and composition according to a typical procedure from the literature (see ESI for detailed synthesis procedure and full characterization) [31]. We confirm the validity of such approach, as this catalyst showed slightly higher performance than TS-1 and TS1_MAC (Figure 4). This results from the increased pore volume $\left(0.41 \mathrm{~cm}^{3} \cdot \mathrm{g}^{-1}\right)$ and external surface area $\left(180 \mathrm{~m}^{2} \cdot \mathrm{g}^{-1}\right)$ owing to the additional presence of mesopores. It should be noted however that this synthesis involves the use of a sacrificial soft templating agent, whereas the SAC method does not require any additional template. Besides, the amount of $\mathrm{TPAOH}$ used here remains quite low $(\mathrm{TPAOH} / \mathrm{Si} \approx$ $0.16)$ compared to other hierarchical TS-1 (TPAOH/Si $=0.21$ for TS-1_MES [31]) and conventional TS-1 $(\mathrm{TPAOH} / \mathrm{Si}=0.41$ [35]). These two characteristics may contribute to reduce the cost of fabrication of the catalyst, which is of outmost importance for the industrial use of TS-1 based catalysts [51].

\section{Conclusion}

Herein, we show that combining titanium and silicon precursors in the preparation of amorphous $\mathrm{Ti}-$ $\mathrm{SiO}_{2}$ nanoparticles followed by steam assisted crystallization in the presence of TPAOH allowed obtaining a micro-/macroporous titanosilicate zeolite with the MFI-type structure. The crystals of this new macro-microporous TS- 1 catalyst are about 15 times larger than the nanosized TS-1 benchmark. Yet their external surface area remained relatively high, owing to the presence of the macropores. This catalyst performed as well as the nanosized TS-1 zeolite in the epoxidation of allyl alcohol in water. Notably, the larger crystal size ensures an easier recovery of the material.

\section{Acknowledgements}

Authors acknowledge the 'Communauté française de Belgique' for the financial support through the ARC programme (15/20-069). F. Devred is acknowledged for the technical and logistical support. V. Smeets is thankful to F.R.S.-F.N.R.S for his FRIA PhD grant. F.R.S.-F.N.R.S is also thanked for the acquisition of the (i) DR UV-visible equipment used (project CDRJ.0156.18, supervisor E. M. Gaigneaux) and of the (ii) X-ray diffraction equipment used (project EQP U.N006.16F, supervisor E. M. Gaigneaux).

\section{References}

[1] M. Taramasso, G. Perego, B. Notari, Preparation of porous crystalline synthetic material comprised of silicon and titanium oxides, US4410501A, 1983. https://patents.google.com/patent/US4410501A/en (accessed August 21, 2018).

[2] M.G. Clerici, P. Ingallina, Epoxidation of Lower Olefins with Hydrogen Peroxide and Titanium Silicalite, Journal of Catalysis. 140 (1993) 71-83. doi:10.1006/jcat.1993.1069.

[3] J. Přech, Catalytic performance of advanced titanosilicate selective oxidation catalysts - a review, Catalysis Reviews. $60 \quad$ (2018) 71-131. doi:10.1080/01614940.2017.1389111.

[4] M. Hartmann, A.G. Machoke, W. Schwieger, Catalytic test reactions for the evaluation of hierarchical zeolites, Chem. Soc. Rev. 45 (2016) 3313-3330. doi:10.1039/C5CS00935A.

[5] V. Smeets, C. Boissière, C. Sanchez, E.M. Gaigneaux, E. Peeters, B.F. Sels, M. Dusselier, D.P. Debecker, Aerosol Route to TiO2-SiO2 Catalysts with Tailored Pore Architecture and High Epoxidation Activity, Chem. Mater. 31 (2019) 1610-1619. doi:10.1021/acs.chemmater.8b04843.

[6] V. Smeets, L. van den Biggelaar, T. Barakat, E.M. Gaigneaux, D.P. Debecker, Macrocellular Titanosilicate Monoliths as Highly Efficient Structured Olefin Epoxidation Catalysts, ChemCatChem. $11 \quad$ (2019) 1593-1597. doi:10.1002/cctc. 201900028.

[7] S. Li, J. Li, M. Dong, S. Fan, T. Zhao, J. Wang, W. Fan, Strategies to control zeolite particle morphology, Chem. Soc. Rev. (2019). doi:10.1039/C8CS00774H.

[8] K. Na, C. Jo, J. Kim, W.-S. Ahn, R. Ryoo, MFI Titanosilicate Nanosheets with Single-Unit-Cell Thickness as an Oxidation Catalyst Using Peroxides, $\begin{array}{lllll}\text { ACS } & \text { Catal. } & 1 & \text { (2011) 901-907. }\end{array}$ doi:10.1021/cs2002143.

[9] J. Přech, P. Eliášová, D. Aldhayan, M. Kubů, Epoxidation of bulky organic molecules over pillared titanosilicates, Catalysis Today. 243 (2015) 134-140. doi:10.1016/j.cattod.2014.07.002.

[10] M.-H. Zhu, L. Li, L. Chen, Y.-X. Liao, W.-J. Ding, Z.-J. Feng, Z. Wan, N. Hu, X.-S. Chen, H. Kita, Preparation of TS-1 zeolite membrane from dilute precursor synthesis solution, Microporous and Mesoporous Materials. $273 \quad$ (2019) 212-218. doi:10.1016/j.micromeso.2018.07.017.

[11] Z. Han, Y. Shen, X. Qin, F. Wang, X. Zhang, G. Wang, H. Li, Synthesis of Hierarchical Titanium-Rich Titanium Silicalite-1 Zeolites and the Highly Efficient Catalytic Performance for Hydroxylation of Phenol, ChemistrySelect. 4 (2019) 1618-1626. doi:10.1002/slct.201803864.

[12] G. Xiong, D. Hu, Z. Guo, Q. Meng, L. Liu, An efficient Titanium silicalite-1 catalyst for propylene epoxidation synthesized by a combination of aerosolassisted hydrothermal synthesis and recrystallization, Microporous and Mesoporous Materials. 268 (2018) 93-99. doi:10.1016/j.micromeso.2018.04.015.

[13] Y. Jiao, A.-L. Adedigba, Q. He, P. Miedziak, G. Brett, N.F. Dummer, M. Perdjon, J. Liu, G.J. Hutchings, Inter-connected and open pore hierarchical TS-1 with 
controlled framework titanium for catalytic cyclohexene epoxidation, Catal. Sci. Technol. 8 (2018) 2211-2217. doi:10.1039/C7CY02571H.

[14] M.B. Yue, M.N. Sun, F. Xie, D.D. Ren, Dry-gel synthesis of hierarchical TS-1 zeolite by using P123 and polyurethane foam as template, Microporous and Mesoporous Materials. 183 (2014) 177-184. doi:10.1016/j.micromeso.2013.09.029.

[15] Y. Zuo, T. Zhang, M. Liu, Y. Ji, C. Song, X. Guo, Mesoporous/Microporous Titanium Silicalite with Controllable Pore Diameter for Cyclohexene Epoxidation, Ind. Eng. Chem. Res. 57 (2018) 512520. doi:10.1021/acs.iecr.7b03719.

[16] S.-K. Kim, B.M. Reddy, S.-E. Park, HighPerformance Microwave Synthesized Mesoporous TS1 Zeolite for Catalytic Oxidation of Cyclic Olefins, Ind. Eng. Chem. Res. 57 (2018) 3567-3574. doi:10.1021/acs.iecr.7b04556.

[17] H. Xin, J. Zhao, S. Xu, J. Li, W. Zhang, X. Guo, E.J.M. Hensen, Q. Yang, C. Li, Enhanced Catalytic Oxidation by Hierarchically Structured TS-1 Zeolite, J. Phys. Chem. C. 114 (2010) 6553-6559. doi:10.1021/jp912112h.

[18] S. Du, X. Chen, Q. Sun, N. Wang, M. Jia, V. Valtchev, J. Yu, A non-chemically selective top-down approach towards the preparation of hierarchical TS-1 zeolites with improved oxidative desulfurization catalytic performance, Chem. Commun. 52 (2016) 3580-3583. doi:10.1039/C5CC10232D.

[19] L.-H. Chen, X.-Y. Li, G. Tian, Y. Li, J.C. Rooke, G.S. Zhu, S.-L. Qiu, X.-Y. Yang, B.-L. Su, Highly Stable and Reusable Multimodal Zeolite TS-1 Based Catalysts with Hierarchically Interconnected ThreeLevel Micro-Meso-Macroporous Structure, Angew. Chem. Int. Ed. 50 (2011) 11156-11161. doi:10.1002/anie.201105678.

[20] W. Cheng, Y. Jiang, X. Xu, Y. Wang, K. Lin, P.P. Pescarmona, Easily recoverable titanosilicate zeolite beads with hierarchical porosity: Preparation and application as oxidation catalysts, Journal of Catalysis. 333 (2016) 139-148. doi:10.1016/j.jcat.2015.09.017.

[21] J. Lin, F. Xin, L. Yang, Z. Zhuang, Synthesis, characterization of hierarchical TS-1 and its catalytic performance for cyclohexanone ammoximation, Catalysis Communications. 45 (2014) 104-108. doi:10.1016/j.catcom.2013.11.005.

[22] Y. Wang, M. Lin, A. Tuel, Hollow TS-1 crystals formed via a dissolution-recrystallization process, Microporous and Mesoporous Materials. 102 (2007) 80-85. doi:10.1016/j.micromeso.2006.12.019.

[23] C. Pagis, A.R. Morgado Prates, D. Farrusseng, N. Bats, A. Tuel, Hollow Zeolite Structures: An Overview of Synthesis Methods, Chem. Mater. 28 (2016) 5205-5223. doi:10.1021/acs.chemmater.6b02172.

[24] Z. Guo, G. Xiong, L. Liu, P. Li, L. Hao, Y. Cao, F. Tian, Aerosol-assisted synthesis of hierarchical porous titanosilicate molecular sieve as catalysts for cyclohexene epoxidation, J Porous Mater. 23 (2016) 407-413. doi:10.1007/s10934-015-0094-7.
[25] L. Lakiss, M. Rivallan, J.-M. Goupil, J. El Fallah, S. Mintova, Self-assembled titanosilicate TS-1 nanocrystals in hierarchical structures, Catalysis Today. $\quad 168 \quad$ (2011) 112-117. doi:10.1016/j.cattod.2010.12.045.

[26] W.J. Kim, T.J. Kim, W.S. Ahn, Y.J. Lee, K.B. Yoon, Synthesis, Characterization and Catalytic Properties of TS-1 Monoliths, Catalysis Letters. 91 (2003) 123127. doi:10.1023/B:CATL.0000006327.29961.26.

[27] H. Liu, G. Lu, Y. Guo, Y. Guo, J. Wang, Deactivation and regeneration of TS-1/diatomite catalyst for hydroxylation of phenol in fixed-bed reactor, Chemical Engineering Journal. 108 (2005) 187-192. doi:10.1016/j.cej.2005.01.011.

[28] K. Möller, B. Yilmaz, R.M. Jacubinas, U. Müller, T. Bein, One-Step Synthesis of Hierarchical Zeolite Beta via Network Formation of Uniform Nanocrystals, J. Am. Chem. Soc. 133 (2011) 5284-5295. doi:10.1021/ja108698s.

[29] W. Song, Z. Liu, L. Liu, A.L. Skov, N. Song, G. Xiong, K. Zhu, X. Zhou, A solvent evaporation route towards fabrication of hierarchically porous ZSM-11 with highly accessible mesopores, RSC Adv. 5 (2015) 31195-31204. doi:10.1039/C5RA02493E.

[30] Y. Zhang, K. Zhu, X. Zhou, W. Yuan, Synthesis of hierarchically porous ZSM-5 zeolites by steamassisted crystallization of dry gels silanized with shortchain organosilanes, New J. Chem. 38 (2014) 58085816. doi:10.1039/C4NJ00811A.

[31] Z. Kong, B. Yue, W. Deng, K. Zhu, M. Yan, Y. Peng, H. He, Direct synthesis of hierarchically porous TS-1 through a solvent-evaporation route and its application as an oxidation catalyst, Appl. Organometal. Chem. 28 (2014) 239-243. doi:10.1002/aoc.3115.

[32] Q. Du, Y. Guo, P. Wu, H. Liu, Y. Chen, Facile synthesis of hierarchical TS-1 zeolite without using mesopore templates and its application in deep oxidative desulfurization, Microporous and Mesoporous Materials. 275 (2019) 61-68. doi:10.1016/j.micromeso.2018.08.018.

[33] A.G. Machoke, A.M. Beltrán, A. Inayat, B. Winter, T. Weissenberger, N. Kruse, R. Güttel, E. Spiecker, W. Schwieger, Micro/macroporous system: MFI-type zeolite crystals with embedded macropores, Adv. Mater. Weinheim. 27 (2015) 1066-1070. doi:10.1002/adma.201404493.

[34] T. Weissenberger, B. Reiprich, A.G.F. Machoke, K. Klühspies, J. Bauer, R. Dotzel, J.L. Casci, W. Schwieger, Hierarchical MFI type zeolites with intracrystalline macropores: the effect of the macropore size on the deactivation behaviour in the MTO reaction, Catal. Sci. Technol. 9 (2019) 32593269. doi:10.1039/C9CY00368A.

[35] A. Thangaraj, M.J. Eapen, S. Sivasanker, P. Ratnasamy, Studies on the synthesis of titanium silicalite, TS-1, Zeolites. 12 (1992) 943-950. doi:10.1016/0144-2449(92)90159-M.

[36] M. Jacquemin, M.J. Genet, E.M. Gaigneaux, D.P. Debecker, Calibration of the X-Ray Photoelectron Spectroscopy Binding Energy Scale for the 
Characterization of Heterogeneous Catalysts: Is Everything Really under Control?, ChemPhysChem. 14 (2013) 3618-3626. doi:10.1002/cphc.201300411.

[37] B.M. Reddy, B. Chowdhury, P.G. Smirniotis, An XPS study of the dispersion of $\mathrm{MoO} 3$ on $\mathrm{TiO} 2-\mathrm{ZrO} 2$, $\mathrm{TiO} 2-\mathrm{SiO} 2, \mathrm{TiO} 2-\mathrm{Al} 2 \mathrm{O} 3, \mathrm{SiO} 2-\mathrm{ZrO} 2$, and $\mathrm{SiO} 2-$ TiO2-ZrO2 mixed oxides, Applied Catalysis A: General. 211 (2001) 19-30. doi:10.1016/S0926860X(00)00834-6.

[38] B. Erdem, R.A. Hunsicker, G.W. Simmons, E.D. Sudol, V.L. Dimonie, M.S. El-Aasser, XPS and FTIR Surface Characterization of $\mathrm{TiO} 2$ Particles Used in Polymer Encapsulation, Langmuir. 17 (2001) 26642669. doi:10.1021/la0015213.

[39] B.S. Shirke, P.V. Korake, P.P. Hankare, S.R. Bamane, K.M. Garadkar, Synthesis and characterization of pure anatase $\mathrm{TiO} 2$ nanoparticles, J Mater Sci: Mater Electron. 22 (2011) 821-824. doi:10.1007/s10854010-0218-4.

[40] A. Kim, C. Sanchez, G. Patriarche, O. Ersen, S. Moldovan, A. Wisnet, C. Sassoye, D. P. Debecker, Selective $\mathrm{CO} 2$ methanation on $\mathrm{Ru} / \mathrm{TiO} 2$ catalysts: unravelling the decisive role of the TiO 2 support crystal structure, Catalysis Science \& Technology. 6 (2016) 8117-8128. doi:10.1039/C6CY01677D.

[41] L.E. Manangon-Perugachi, A. Vivian, P. Eloy, D.P. Debecker, C. Aprile, E.M. Gaigneaux, Hydrophobic titania-silica mixed oxides for the catalytic epoxidation of cyclooctene, Catalysis Today. (2019). doi:10.1016/j.cattod.2019.05.020.

[42] A.M. Cojocariu, P.H. Mutin, E. Dumitriu, F. Fajula, A. Vioux, V. Hulea, Mild oxidation of bulky organic compounds with hydrogen peroxide over mesoporous TiO2-SiO2 xerogels prepared by non-hydrolytic solgel, Applied Catalysis B: Environmental. 97 (2010) 407-413. doi:10.1016/j.apcatb.2010.04.027.

[43] G. Berlier, V. Crocellà, M. Signorile, E. Borfecchia, F. Bonino, S. Bordiga, Characterization of Metal Centers in Zeolites for Partial Oxidation Reactions, in: J. Pérez Pariente, M. Sánchez-Sánchez (Eds.), Structure and Reactivity of Metals in Zeolite Materials, Springer International Publishing, Cham, 2018: pp. 91-154. doi:10.1007/430_2018_24.

[44] K. Madhusudan Reddy, S.V. Manorama, A. Ramachandra Reddy, Bandgap studies on anatase titanium dioxide nanoparticles, Materials Chemistry and Physics. 78 (2003) 239-245. doi:10.1016/S02540584(02)00343-7.

[45] J.C. Groen, L.A.A. Peffer, J. Pérez-Ramírez, Pore size determination in modified micro- and mesoporous materials. Pitfalls and limitations in gas adsorption data analysis, Microporous and Mesoporous Materials. 60 (2003) 1-17. doi:10.1016/S1387-1811(03)00339-1.

[46] J.P. Thielemann, F. Girgsdies, R. Schlögl, C. Hess, Pore structure and surface area of silica SBA-15: influence of washing and scale-up, Beilstein Journal of Nanotechnology. 2 (2011) 110-118. doi:10.3762/bjnano.2.13.

[47] G. Deo, A.M. Turek, I.E. Wachs, D.R.C. Huybrechts, P.A. Jacobs, Characterization of titania silicalites,
Zeolites. 13 (1993) 365-373. doi:10.1016/01442449(93)90151-R.

[48] V. Smeets, L. Ben Mustapha, J. Schnee, E.M. Gaigneaux, D.P. Debecker, Mesoporous SiO2-TiO2 epoxidation catalysts: Tuning surface polarity to improve performance in the presence of water, Molecular Catalysis. 452 (2018) 123-128. doi:10.1016/j.mcat.2018.04.011.

[49] M.G. Clerici, The activity of titanium silicalite-1 (TS1): Some considerations on its origin, Kinet Catal. 56 (2015) 450-455. doi:10.1134/S0023158415040059.

[50] Y. Zuo, M. Liu, T. Zhang, C. Meng, X. Guo, C. Song, Enhanced Catalytic Performance of Titanium Silicalite-1 in Tuning the Crystal Size in the Range 1200-200 $\mathrm{nm}$ in a Tetrapropylammonium Bromide System, ChemCatChem. 7 (2015) 2660-2668. doi:10.1002/cctc.201500440.

[51] G. Ming-Xing, G. Hong-Chen, W. Xiang-Sheng, G. Wei-Min, Gas Phase Epoxidation of Propylene with $\mathrm{O} 2$ Induced by Alternating Electric Field, Chinese Journal of Chemistry. 23 (2005) 471-473. doi:10.1002/cjoc.200590471. 